\title{
Calculating algorithm of service quality statistical parameters for asynchronous network subscribers
}

\author{
Kemelbekova Zh. S., Sembiyev O. Z., Umarova Zh. R.
}

M. Auezov South-Kazakhstan State University, Kazakhstan

\begin{tabular}{|c|c|}
\hline Article Info & ABSTRACT \\
\hline Article history: & \multirow{10}{*}{$\begin{array}{l}\text { It is often necessary to determine statistical parameters that characterize } \\
\text { the quality of service on the network by managing when designing computer } \\
\text { networks using the concept of virtual connections with bypass directions. } \\
\text { In many ways, the attainable level of quality of the services provided is } \\
\text { determined at the stage of network design, when decisions was made } \\
\text { regarding the subscriber capacity of stations, the capacity of bundles of trunk } \\
\text { lines, the composition and volume of telecommunication services provided. } \\
\text { Despite constant progress in the field of network technologies, the problem } \\
\text { of determining the necessary amount of network resources and ensuring } \\
\text { the quality of user service remains relevant. In this regard, this article } \\
\text { discusses a broadband digital network with service integration, based on an } \\
\text { asynchronous network in which an iterative method implemented. } \\
\text { Here the flow distribution is determined by the route matrix, and the load } \\
\text { distribution between the nodes of each pair of nodes made through the path } \\
\text { tree obtained on the matrix of routes when calculating this pair. At the same } \\
\text { time, an algorithm has been built for allow optimal allocation of channel } \\
\text { resources between circuit switching and packet switching subnets within an } \\
\text { asynchronous network. }\end{array}$} \\
\hline Received Jan 25, 2020 & \\
\hline Revised May 1, 2020 & \\
\hline Accepted May 20, 2020 & \\
\hline Keywords: & \\
\hline Algorithm & \\
\hline Asynchronous network & \\
\hline Channel switching & \\
\hline Computer networks & \\
\hline Packet switching & \\
\hline
\end{tabular}

\section{Corresponding Author:}

Kemelbekova Zhanar,

Natural Science and Pedagogical Higher School,

M.Auezov South-Kazakhstan State University, Kazakhstan.

Email: kemel_zhan@mail.ru

\section{INTRODUCTION}

In the process of network analysis and synthesis, the problem of calculating the quality of service parameters of an asynchronous network is a greatest interest for designers, since the solution to this problem can significantly improve network performance, prevent network failures during network congestion, and determine optimal bypass directions for traffic transmission. The design calculation of the quality of service parameters of an asynchronous network is necessary, since the complexity of its configuration, as well as its operation in real time and in interactive mode, greatly reduces the ability to intuitively evaluate the performance of an asynchronous network. The inability to achieve design capacity in a real-life asynchronous network is due to a lack of understanding at the design stage of the characteristics of the future workload and its interaction with system components [1-8].

The study of this problem seems important because the models developed in the work of calculating the quality of service parameters of an asynchronous network allow adequately display the interaction between the workload and network resources and evaluate its expected performance. Using the developed information system, critical, peak situations have been prevented that inevitably arise during network congestion. This method allows tracking the occurrence of such unwanted situations and eliminating their consequences. This is achieved mainly through the efficient use of bypass directions of load transfer. 
At the same time, the time of transmission and delivery of data packets is reduced, which significantly affects the quality of the information stream transported via digital communication channels $[9,10]$.

Currently, most methods for assessing the quality of user service on communication networks used in the design either consider the problem in relation to one-dimensional traffic or do not take into account the influence of a finite number of load sources [11-14]. This limits methods using in solving design problems of multiservice networks, including in areas of subscriber access networks [15, 16]. The solution of these problems is impossible without scientifically based design methods. Therefore, this article is devoted to the research and development of a method for calculating the quality of user service when accessing multiservice networks, taking into account multidisciplinary traffic, the factor of a finite number of sources and the features of constructing an access section on modern multiservice networks [17-20].

\section{RESEARCH METHOD}

When developing a model for calculating the statistical parameters of the quality of service of an asynchronous network (ATM), will be assumed that this network is mainly intended for transporting multimedia traffic via communication channels, that is, mainly for transmitting video and audio information. This type of information uses the channel switching method, namely, before betraying this information, it is necessary to connect virtual channels between the corresponding subscribers of the network [21-25]. This connection of virtual paths is provided using special packages called multi-channel calls. In this regard, for a broadband digital network with service integration (B-DNSI) based on ATM, most of the cycle time positions of the pulse-code modulation (PCM) $90 \%$ are allocated for transmitting information in channel switching mode and the PCM cycle boundary for the session remains unchanged. Therefore, the main object of the ATM network research is the QC subnet with bypass directions. In the described model, it is considered as a system with obvious losses, that is, when the system is blocked, calls coming into it are irretrievably lost [26-28]. In CS networks, as an objective function in the problem of determining the optimal throughput, as a rule, the average message losses in the network or the average network probability of a denial of service are selected. These criteria ensure maximum use of network bandwidth and since their average values are calculated, therefore these parameters are considered statistical [29].

The quality of service on the SC subnet is usually estimated by the probabilities of losses on the network branches. It is known that the quality of service for incoming calls characterized by the possibility of connections or the length of time that connections are provided. There are two main methods, two disciplines of servicing incoming calls: lossless and loss. Lossless service is the discipline in which an incoming call served immediately, and with loss, if the incoming call receives either a denial of service or its service is delayed for some time. For economic reasons, real switching systems are designed with losses usually. In this case, a system with obvious losses is considered. Serving with obvious losses is a discipline in which a call arriving at a switching system, receiving a denial of service, leaves the system and no longer influences the system. With this discipline of service, the subscriber, having received a refusal signal to establish a connection with the destination host, refuses further attempts to establish this connection [30-35].

$\lambda_{j}$ - the intensity of incoming calls, $\Pi$ - a system service device that processes connection call requests, $\mu_{j}$ - modules that process incoming confirmations, $K-$ a switch that distributes calls among modules. Modules $\Pi$ and $\mu_{j}$ are logical elements, the implementation methods of which depend on the applied hardware and software.

In this case, the first method of the model of hybrid switching nodes (HSN) can represented as a queuing system (QS) of the type $M / M / 1$ that is, having one serving device. This representation differs from the model of nodes used in the design of information and computer systems (CS), in which either the processing time in the first phase neglects, or the models describe the processing processes in nodes represented in the form of multiprocessor devices. The processing time of packets in the first phase couldn't neglected due to the rather lengthy operations that can be carried out at the first stage of processing service packets of the "connection request" type $[36,37]$. An analysis of the functioning process carries out under the following assumptions.

1) A Poisson stream of connection requests with intensity $\lambda_{j}$ arrives at the user node.

2) The lengths of service packets representing connection requests are determined independently randomly. It can assume that the packet length is distributed exponentially with the average $1 / \mu_{j}$, where is $\mu_{j}$ the call service intensity. 
Ergodicity of such a call service system takes place provided that $\rho=\lambda_{j} / \mu_{j}<1$.

The quality of service on the CS subnet, presented as a service system with obvious losses. This is estimated by the probability of a subscriber who refuses to establish a connection with the destination host for further transmission of information traffic [38, 39]. As the CS subnet stream, can be considered the call flow entering the ATM network to the $i$ sender node and destined for the $j$ address node. In general, an address is a coded designation of a point of departure or destination of data. The address of the object is determined by the number, code and phrase. The list of objects includes registers, memory cells, external devices, communication channels, processes, systems, networks. Recipient data objects are commonly referred to as recipients. Often the address is associated with the name of the object [40].

Let $r_{i}(j)$ - be the average intensity of the multichannel call (MC) flow, entering the ATM network at the $i$ sending node and destined for the $j$ addressing node. The value $r_{i}(j)$ will be called the input load of the ATM network. It represents the average value of the incoming load between the corresponding pairs of nodes per hour of least load.

Let $t_{i}(j)$ - be the average intensity of the total flux of MC passing through the $i$ node and intended for the $j$ node. The value $t_{i}(j)$ will be called the nodal load of the ATM network. It includes both the $r_{i}(j)$ input load and the $t_{l}(j)$ loads entering the $i$ node from all $l$ nodes adjacent to it. The order of selection of the outgoing directions from the $i$ node for transferring the $t_{i}(j)$ load to all other neighboring nodes, i.e. its distribution plan $[41,42]$ is represented by the $M$ route matrix for the $i$ node.

$$
M_{i}=\begin{gathered}
i k_{1} \\
\cdot \\
\cdot i k_{S_{i}}
\end{gathered}\left[\begin{array}{cccc}
1 & 2 & \cdots & \mathrm{n} \\
\mu_{i k_{1}, 1} & \mu_{i k_{1}, 2} & \cdots & \mu_{i k_{1}, n} \\
\mu_{i k_{2}, 1} & \mu_{i k_{2}, 2} & \cdots & \mu_{i k_{2}, n} \\
\cdot & \cdot & \cdots & \cdot \\
\mu_{i k_{S_{i}}, 1} & \mu_{i k_{S_{i}}, 1} & \cdots & \mu_{i k_{S_{i}}, n}
\end{array}\right]
$$

In the route matrix, the number of columns is equal $n-1$ (there is no column in the $M_{i}$ matrix for $\mathrm{i}$ node), and the number of rows - the number of $S_{i}$ nodes incident with i node. The element $\mu_{i k_{s_{i}}, j}$ of the $M_{i}$ matrix is the number of the order in which the $\left(i k_{S_{i}}\right)$ branch is selected when establishing a connection to the node $j$, i.e. $\mu_{i k_{s_{i}}, j} \in\left\{1,2, \ldots . S_{i}\right\}$. If a set of routing matrices is specified $\left\{M_{i}, i=1, n\right\}$, then this means that an information distribution plan is set for the entire QC subnet. With a static plan for the distribution of information, static (fixed) routing is carried out in the CS subnet [43, 44]. The amount of missed or excess load depends on the probability of loss of $t_{i}(j)$ traffic allocated to the $(i k)$ branch. Let introduce the following notation

$$
h_{i k}(j)=\varphi_{i k}(j)\left[1-p_{i k}(j)\right] ; \quad \forall i, k, j \in \mathrm{V},
$$

where $h_{i k}(j) \in[0 ; 1)$ - is a characteristic of an $(i k)$ missed branch of $t_{i}(j)$ load branch. The function $h_{i k}(j)$ is the conditional probability of the $t_{i}(j)$ load passing through the $(i k)$ branch when the service is busy with all the directions preceding this branch. In the future, $h_{i k}(j)$ will be called the probability of servicing the $t_{i}(j)$ load by the $(i k)$ branch, and the $h_{i k}(j) * t_{i}(j)$ value - the $(i k)$ load missed by the branch.

Let be $r_{i}^{u}(j)$ - the input load of the $G^{u}(j)$ path tree. Let us define the $t_{i}^{u}(k, j)$ as transit load on the $i$ node, which forms during the distribution of the $r_{i}^{u}(j)$ input load along the branches of the $G^{u}(j)$ 
path tree and passing through the $i$ node adjacent to the $k \in K^{u}(j)$ node [45]. Finding transit nodes on each node of the tree is carried out using the following formula,

$$
t_{i}^{u}(k, j)=t_{l}^{u}(i, j) \cdot h_{l i}(j)
$$

where $(l i) \in L^{u}(j), i, j, k, l \in V^{u}(j)$. Without loss of generality, we assume that for all nodes of the $i=u$ path tree, $t_{i}^{i}(k, j)=r_{i}^{u}(j)$ value, and for $i, k=j$ nodes, $t_{j}^{u}(j, j)=t_{j}^{u}(j)$ value.

It should be noted that since any node of the path tree, except the initial one, contains only one branch, then on this node, regardless of the choice of directions coming from it, the values of all transit loads will be the same. Thus, for any $i \in V^{u}(j)$ node $(i \neq u)$, and all $k_{m} \in K^{u}(j), m=1,2, \ldots S$ nodes, the relation

$$
t_{i}^{u}\left(k_{1}, j\right)=t_{i}^{u}\left(k_{2}, j\right)=\ldots=t_{s}^{u}\left(k_{1}, j\right)=r_{i}^{u}(j)
$$

Theorem 1. For all $(i k) \in L^{u}(j)$ branches, the formula

$$
\sum_{k \in K_{i}^{u}(j)} h_{i k}(j)=1-\Pi_{i}^{u}(j)
$$

Using the Theorem 1 for a given $H=\left\{h_{i k}(j)\right\}$ set at each node of the path tree, all current values of the loss probabilities are calculated. However, it is more convenient to determine the share of the load lost in the path tree by sequentially summing the parts of the load lost in the transit nodes of this tree. The probability of losses between a pair of nodes $i$ and $j$ is defined as the ratio of the load lost at all nodes of the tracks to the received one [46]. In this case, the probability of losses for the path tree between $i$ and $j$ nodes will be

$$
P_{i}^{u}(j)=\frac{\sum_{s \in V^{u}(j)} r_{s}^{u}(j) \Pi_{s}(j)}{r_{i}^{u}(j)}
$$

where $V^{u}(j)$ - a subset of all nodes of the path tree between nodes $i$ и $j$.

To calculate the probability of blocking or loss of a message from using an iterative algorithm, the main performance characteristics are estimated relative to those obtained for the case with a simple static setting of the throughput, if the average channel throughput in both cases is the same [47]. The value obtained because of this algorithm corresponds to estimates, which confirms the correctness of the calculations.

An iterative algorithm for calculating the quality of service parameters is presented, which involves the following operations:

1) Selection of a pair of nodes with nonzero gravity;

2) Building a tree of paths by route matrices for the selected pair of nodes;

3) Calculation of the total load on each branch of the path tree. When calculating the load, the probability of losses on the remainder of the path tree from the transit node to the destination node is taken into account [48]. At the beginning of the algorithm, all probability of loss on the branches are assumed equal to 0;

4) Determination of the probability of losses by the formula (5) for the pair in question as the ratio of the lost load to the received;

5) Operations 1-4 are performed for all gravitating pairs in the network in question, as a result of which the total load on each branch from all pairs of nodes and the loss matrix between pairs of nodes will be obtained; 
6) Determination of the maximum deviation between the probability of losses $P_{i}^{q-1}(j)$ between a pair of nodes $\mathrm{i}$ and $\mathrm{j}$, obtained in the previous iteration, and $P_{i}^{q}(j)$, obtained in this iteration, according to the formula $\delta=\max _{i, j}\left|P_{i}^{q}(j)-P_{i}^{q-1}(j)\right|$;

7) Comparison $\delta$ with a pre-selected value $\varepsilon$. If $\delta>\varepsilon$, then from the values of the total load for each branch, the probability of losses on the branch and the transition to the next iteration is determined. If $\delta \leq \varepsilon$, then another iteration is performed and the implementation of the algorithm ends

Block diagram of the algorithm as shown in Figure 1-2.

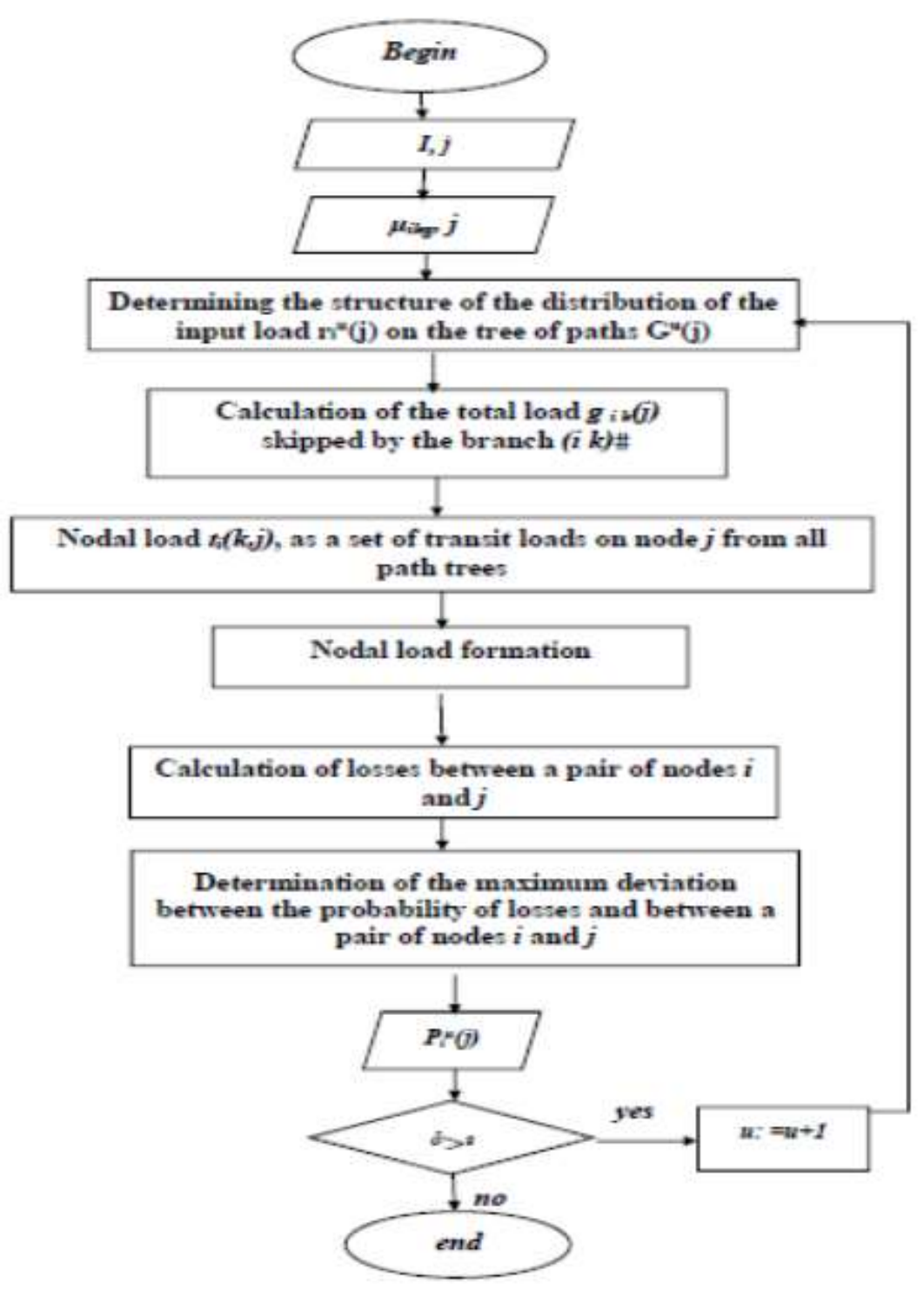

Figure 1. Block diagram of the algorithm 


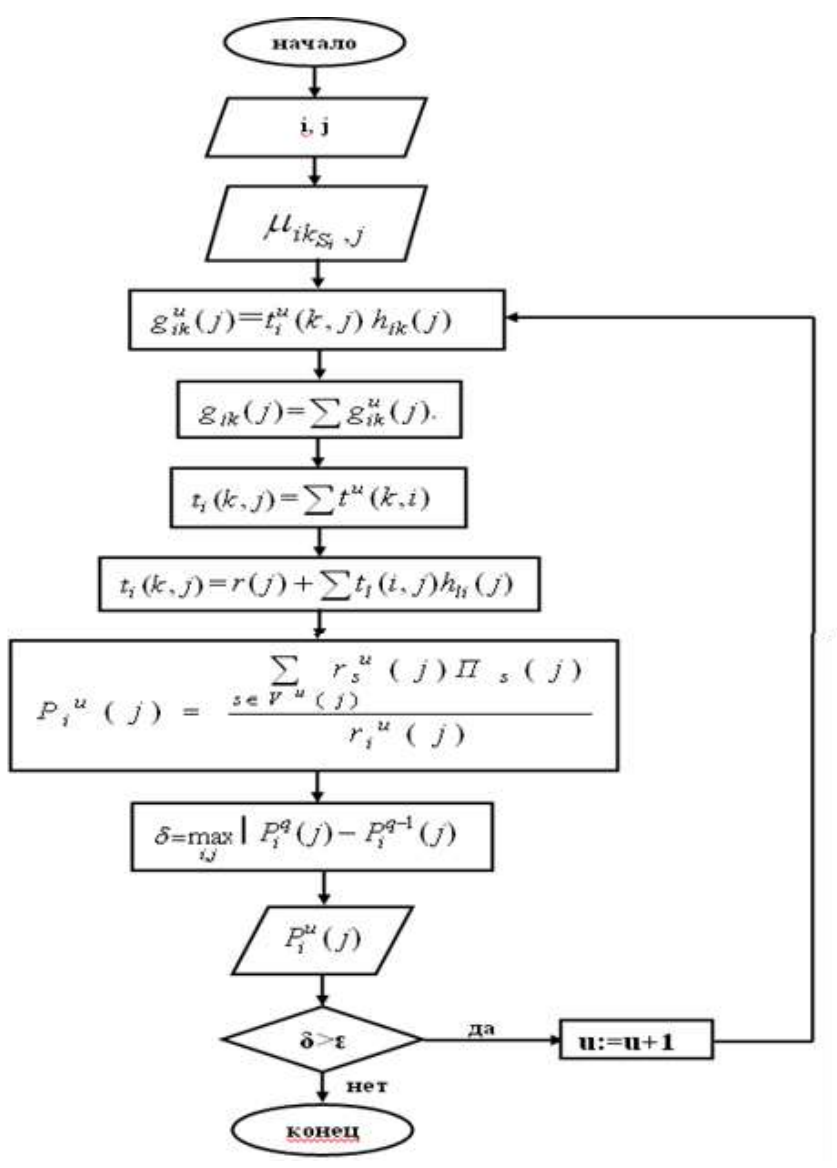

Figure 2. Block - algorithm diagram (in the form of a formula)

\section{RESULTS AND DISCUSSION}

We show the implementation of the algorithm on a specific numerical example. Let B-DNSI with six hybrid-switching nodes, presented in the form of a directed graph in Figure 3. Circles indicate the numbers of the IGP (integral group path). Throughput and the number of channels for each path is $G_{j}$ $=10 \mathrm{bit} / \mathrm{sec} . N_{j}=50$. The service time of one call to the SC network is $1 / G_{j} v_{j} j=60 \mathrm{sec}$. The total allowable losses $\pi_{0}=40$ Erl.

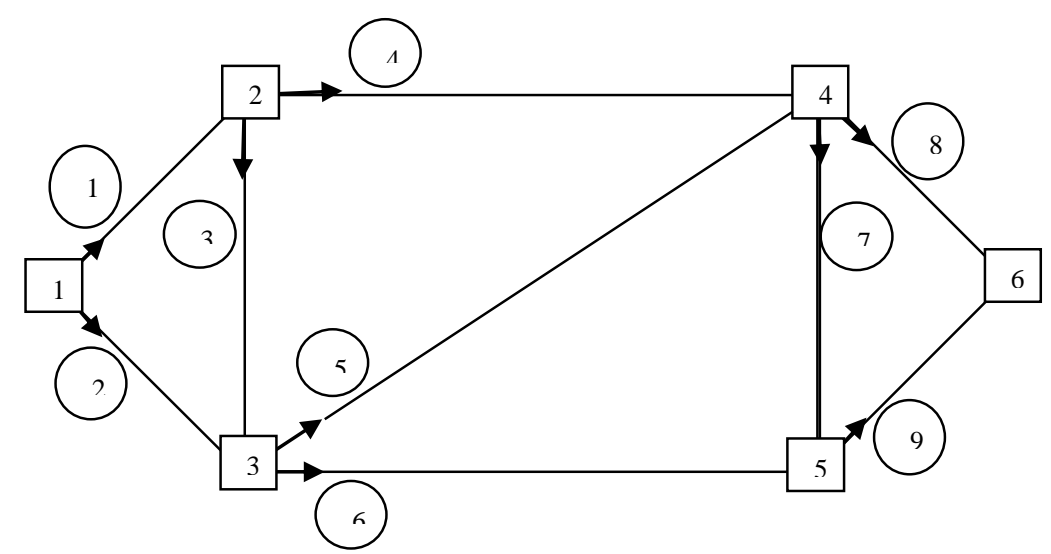

Figure 3. ATM network graph with six hybrid-switching nodes 
Calculation data for CS subnet (Table 1).

Table 1. Initial input traffic (messages per second):

\begin{tabular}{ccccccc}
\hline $\mathrm{F}^{1}$ & 1 & 2 & 3 & 4 & 5 & 6 \\
\hline 1 & - & 0.507 & 0.3 & 0.1 & 0.08 & 0.1 \\
2 & - & - & 0.6 & 0.045 & 0.07 & 0.06 \\
3 & - & - & - & 0.567 & 0.083 & 0.1 \\
4 & - & - & - & - & 0.708 & 0.095 \\
5 & - & - & - & - & - & 0.642 \\
6 & - & - & - & - & - & - \\
\hline
\end{tabular}

The specified routing for the main directions is designated: traffic $t_{12}-1 \rightarrow 2$ (from the first node to the second)

$$
\begin{aligned}
& t_{13}-1 \rightarrow 3, t_{14}-1 \rightarrow 3 \rightarrow 4, t_{15}-1 \rightarrow 3 \rightarrow 5, t_{16}-1 \rightarrow 2 \rightarrow 4 \rightarrow 6 \\
& t_{23}-2 \rightarrow 3, t_{24}-2 \rightarrow 4, t_{25}-2 \rightarrow 3 \rightarrow 5, t_{26}-2 \rightarrow 4 \rightarrow 6 \\
& t_{34}-3 \rightarrow 4, t_{35}-3 \rightarrow 5, t_{36}-3 \rightarrow 5 \rightarrow 6 \\
& t_{45}-4 \rightarrow 5, t_{46}-4 \rightarrow 6 \\
& t_{56}-5 \rightarrow 6
\end{aligned}
$$

Total flows:

$$
\begin{aligned}
& \lambda_{1}=f_{16}+f_{12}=0.1+0.507=0.607 \\
& \lambda_{2}=f_{15}+f_{14}+f_{13}=0.49 \\
& \lambda_{3}=f_{25}+f_{23}=0.67 \\
& \lambda_{4}=f_{16}+f_{24}+f_{26}=0.205 \\
& \lambda_{5}=f_{14}+f_{34}=0.667 \\
& \lambda_{6}=f_{15}+f_{25}+f_{36}+f_{35}=0.333 \\
& \lambda_{7}=f_{45}=0.708 \\
& \lambda_{8}=f_{16}+f_{46}+f_{26}=0.255 \\
& \lambda_{9}=f_{36}+f_{56}=0.742
\end{aligned}
$$

Calculation data for the SC subnet (Table 2)

Table 2. Initial input traffic (messages per second):

\begin{tabular}{cccccccc}
\hline $\mathrm{F}^{2}$ & 1 & 2 & 3 & 4 & 5 & 6 & Sum \\
\hline 1 & - & 0.2 & 0.7 & 1.3 & 0.5 & 4.7 & 4.7 \\
2 & - & - & 1.2 & 1.0 & 0.8 & 0.06 & 4.5 \\
3 & - & - & - & 0.567 & 0.083 & 0.1 & 2.1 \\
4 & - & - & - & - & 0.708 & 0.095 & 3.4 \\
5 & - & - & - & - & - & 0.642 & 0.3 \\
6 & - & - & - & - & - & - & $\gamma=15.0$ \\
\hline
\end{tabular}

The specified routing of the main directions.

$$
\begin{aligned}
& t_{12}-1 \rightarrow 2 \\
& t_{13}-1 \rightarrow 3, t_{14}-1 \rightarrow 2 \rightarrow 4, t_{15}-1 \rightarrow 3 \rightarrow 5, t_{16}-1 \rightarrow 3 \rightarrow 5 \rightarrow 6 \\
& t_{23}-2 \rightarrow 3, t_{24}-2 \rightarrow 4, t_{25}-2 \rightarrow 3 \rightarrow 5, t_{26}-2 \rightarrow 4 \rightarrow 6 ; \\
& t_{34}-3 \rightarrow 4, t_{35}-3 \rightarrow 5, t_{36}-3 \rightarrow 4 \rightarrow 6 ;
\end{aligned}
$$


$t_{45}-4 \rightarrow 5, t_{46}-4 \rightarrow 6$

$t_{56}-5 \rightarrow 6$.

Total flows:

$\gamma_{1}=f_{14}+f_{12}=2.2$

$\gamma_{2}=f_{15}+f_{16}+f_{13}=2.5$

$\gamma_{3}=f_{23}+f_{25}=2.0$

$\gamma_{4}=f_{14}+f_{24}+f_{26}=4.5$

$\gamma_{5}=f_{25}+f_{34}=0.667$

$\gamma_{6}=f_{15}+f_{25}+f_{16}+f_{35}=0.333$

$\gamma_{7}=f_{45}=1.8$

$\gamma_{8}=f_{16}+f_{46}+f_{26}=4.2$

$\gamma_{9}=f_{16}+f_{56}=0.8$

The average packet length in the IGP (bit) as shown in Table 3 and 4 .

$1 / \mu_{1}=(0.2 * 100+2 * 80) /(0.2+2.0)=82$

$1 / \mu_{2}=94$

$1 / \mu_{3}=96$

$1 / \mu_{4}=91$

$1 / \mu_{5}=91$

$1 / \mu_{6}=100$

$1 / \mu_{7}=87$

$1 / \mu_{8}=80$

$1 / \mu_{9}=120$

Table 3. Average length of transmitted packets between nodes (bits)

\begin{tabular}{ccccccc}
\hline & 1 & 2 & 3 & 4 & 5 & 6 \\
\hline 1 & - & 100 & 100 & 80 & 80 & 120 \\
2 & - & - & 80 & 100 & 120 & 100 \\
3 & - & - & - & 120 & 100 & 80 \\
4 & - & - & - & - & 80 & 80 \\
5 & - & - & - & - & - & 120 \\
6 & - & - & - & - & - & -
\end{tabular}

Table 4. Calculation of statistical parameters of the quality of service B-DNSI on ATM

\begin{tabular}{ccccccc}
\hline$i$ & $\mathrm{j}$ & $g_{i k}^{u}(j)$ & $g_{i k}(j)$ & $t_{i}(k, j)$ & $\Pi_{i}^{u}(j)$ & $\mathrm{p}_{\mathrm{j}}$ \\
\hline 1 & 2 & 0.507 & 1.653 & 1.507 & 0.614 & 0.1278 \\
1 & 3 & 1.44 & 2.533 & 1.97 & 0.135 & 0.0888 \\
1 & 4 & 1.24 & 2.4203 & 2.67 & 0.0203 & 0.2343 \\
\hline
\end{tabular}

\section{CONCLUSION}

To verify the results using simulation on computers, an experiment was performed to analyze the effectiveness of the algorithm for calculating the quality of service parameters of an asynchronous network. The source of service quality in this experiment is the probability of message loss from real network traffic implementations. The results obtained from the simulation confirm the conclusions about the unconditional increase in the efficiency of the system due to the use of the method of calculating 
the parameters characterizing the quality of service. Thus, an algorithm for calculating the parameters of the quality of service of a broadband network with the integration of services based on an asynchronous network was constructed.

\section{ACKNOWLEDGEMENTS}

A study conducted in the framework of a research project with the financial support of the committee of science of the ministry of education and science of the Republic of Kazakhstan (contract №164-20 of 15.03.18).

\section{REFERENCES}

[1] V. Olifer, "Computer networks. Principles, technologies, protocols: A textbook for university students," St. Petersburg, Peter, pp. 1-944, 2012.

[2] V. Olifer and N. Olifer, "New technologies and equipment of IP-networks," pp. 1-512, 2000.

[3] Goldstein B. S., et al., "Communication networks," St. Petersburg: BHV - Petersburg, pp. 1-400, 2014.

[4] Gordienko V. N., "Multichannel telecommunication systems: Textbook for universities," RiS, pp. 1-396, 2015.

[5] D. Krouse, "Computer networks, Top-down approach," Eksmo, pp. 1-912, 2016.

[6] A. Kemos and A. Zaganiaris, "Economic Prospects of Advanced Telecommunications Services," The Journal of the Institution of British Telecommunications Engineers, vol. 2, no. 3, pp. 38-42, 2001.

[7] E. Pinsky, "Simple approximation for the Erlang loss function," Performance evaluation, vol. 5, no. 3, pp. 155-161, 1992.

[8] Pratt C. W., "The concept of marginal overflow in alternate routing networks," 5 ITC, New-York, pp. 51-58, 1967.

[9] Crook B. I., "Telecommunication systems and networks," vol. 2, GLT, pp. 1-647, 2005.

[10] Markov N. G., "Intelligent navigation and telecommunication control systems for moving objects using cloud computing technology," RiS, pp. 1-158, 2014.

[11] M. Hussaini, et al., "Producer Mobility Support Schemes for Named Data Networking: A Survey," International Journal of Electrical and Computer Engineering (IJECE), vol. 8, no. 6, pp. 5432-5442, 2018.

[12] F. Muzafarov and A. Eshmuradov, "Wireless sensor network based monitoring system for precision agriculture in Uzbekistan," TELKOMNIKA, vol. 17, no. 3, pp. 1071-1080, 2019.

[13] V. S. Lagutin and V. O. Kostrov, "Evaluation of throughput characteristics of multiservice packet networks in the implementation of load type separation technology," Telecommunications, no. 3, pp. 28-32, 2003.

[14] V. S. Lagutin and V. O. Kostrov, "A formalized representation of the process of occupying the transmission band in multiservice packet networks," Telecommunications, no. 1, pp. 31-34, 2003.

[15] Shepel V. M., "Computing systems, networks and telecommunications: Textbook," Finance and Statistics, pp. 1-736, 2008.

[16] Nazarov A. N. and Simonov M. V., “ATM technology of high-speed networks,” ECO-TRENDZ, pp. 1-232, 1997.

[17] Sembiev O. Z. and Kemelbekova Zh. S., "Digital technologies of Kaazakhstan," Materials of the IV international scientific and practical conference Informatics and applied mathematics, Almaty, vol. 1, pp. 72-81, 2019.

[18] Sembiev O. Z., et al., "Load distribution in the mode of switching channels in nodes of asynchronous transport network," Bulletin of Kaznitu, vol. 6, no. 130, pp. 139-146, 2018.

[19] Kemelbekova Zh. S., et al., "Model of transmission of information traffic of multi-channel calls on asynchronous networks," Bulletin of Kaznitu, vol. 1, no. 131, pp. 411-416, 2019.

[20] Kemelbekova Zh. S., et al., "Method of forming a node load in the mode of switching channels of the ATM network," Bulletin of Science of southern Kazakhstan, vol. 3, no. 3, pp. 67-72, 2018.

[21] Ananyev A. N., "Design of multiservice corporate networks of regional telecom operators," Radio and communications, pp. 1-92, 2002.

[22] Bokker P., "Digital network with service integration," Concepts, methods, systems, Radio and communications, pp. 1-304, 1991.

[23] Tishchenko A. B., "Multichannel telecommunication systems," Part 1. The principles of construction of telecommunication systems with a temporary separation of channels: a manual. EC RIOR, SIC INFRA-M, pp. 1-104, 2013.

[24] L. P. Gudyno, "Computing systems, networks and telecommunications: Textbook," KnoRus, pp. 1-376, 2013.

[25] Yershov V. A., "Dispersion estimates of the load of switching nodes," Proceedings of the V all-Union Symposium on control problems on network and communication nodes, Moscow, Nauka, pp. 55-57, 1985.

[26] Yershov V. A. and Yershov D. M. V., "Management of CSIS channel resources on the basis of its reservation," Telecommunications, no. 12, pp. 8-12, 1994.

[27] Yershov V. A. and Kuznetsov N. A., "Method of calculating the throughput capacity of multiservice telecommunication networks," Moscow, Trudy MAC, N1, pp. 22-24, 1999.

[28] Golyshko A. V., et al., "Method of calculating the capacity of the modem pool of Internet service providers," Vestnik svyazi, no. 8, pp. 51-54, 2001.

[29] Tsybakov V. I., "Numerical studies of load dispersion properties," Vestnik svyazi, no. 12, pp. 55-59, 2002.

[30] Tanenbaum E., "Computer networks," Third edition, St. Petersburg, Peter, pp. 1-848, 2002.

[31] N. Olifer and V. Olifer, "Computer Networks: Principles, Technologies and Protocols for Network Design," Wiley \& Sons, England, pp. 1-1000, 2005. 
[32] Filimonov A. Y., "Construction of multiservice Ethernet networks,” SPb. BHV-Petersburg, pp. 1-277, 2007.

[33] Sovetov B. Y. and Yakovlev S. A., "Building Integrated Services Networks," L.: Engineering, pp. 1-332, 1990.

[34] Ginsburg D., "ATM solution for networks working," Addison - Wesley, pp. 1-569, 1996.

[35] A. V. Kuzin, “Computer Networks: A Training Manual,” M.: Forum, SIC INFRA-M, pp. 1-192, 2013.

[36] Kleinrock J. I., "Queuing theory,” M.: Mechanical Engineering, pp. 1-432, 1979.

[37] Vishnevsky V. M., "Theoretical foundations of the design of computer networks," M.: Technosphere, pp. 1-512, 2003.

[38] Kartashevsky V. G., "The basics of queuing theory,” M.: Radio and communications, pp. 1-107, 2006.

[39] Ponomarev D. Y., "Probabilistic temporal characteristics of asynchronous information networks, taking into account self-similarity," M.: Krasnoyarsk, pp. 1-194, 2002.

[40] Roslyakov A. V., "Mathematical models of call centers," M.: IRIAS, pp. 1-336, 2006.

[41] Kemelbekova Zh., et al., "Designing an asynchronous network to compute the quality of telecommunications channels," Proceedings V International scientific practical conference "Industrial technologies and engineering" dedicated to the $75^{\text {th }}$ anniversary of M.Auezov South Kazakhstan State university and $90^{\text {th }}$ anniversary of academician Sultan Tashirbayevich Suleimenov Holding within 4.0 Industrial Revolution, pp. 41-47, 2018.

[42] O. Z. Sembiyev, et al., "Calculations of excess load on the network," News of the national academy of sciences of the Republic of Kazakhstan series of geology and technical sciences, vol. 6, no. 438, pp. 246-255, 2019.

[43] J. Kurose and K. Ross, "Computer Networking: A Top Down Approach," 5th edition, Addison - Wesley, pp. 1-862, 2009.

[44] Sembiev O. Z. and Kemelbekova Zh. S., "Calculation of load distribution on nodes of an asynchronous network," International journal of applied and fundamental research, no. 4, pp. 49-53, 2019.

[45] Sembiev O. Z. and Kemelbekova Zh. S., "Building a broadband digital network architecture with service integration," Proceedings of the XII international conference on applied mathematics and mechanics in the aerospace industry (NPNJ' 2018), Alushta (Crimea), Russia, pp. 710-711, 2018.

[46] Kleinrock L., “Computing systems with queues,” M.: Mir, pp. 1-600, 1979.

[47] Gnedenko B. V. and Kovalenko I. N., "Introduction to queuing theory," M.: Publishing House "Science", pp. 1-432, 1966.

[48] N. G. Kuzmenko, "Computer networks and network technologies," St. Petersburg: Science and Technology, pp. 1-368, 2013.

\section{BIOGRAPHIES OF AUTHORS}
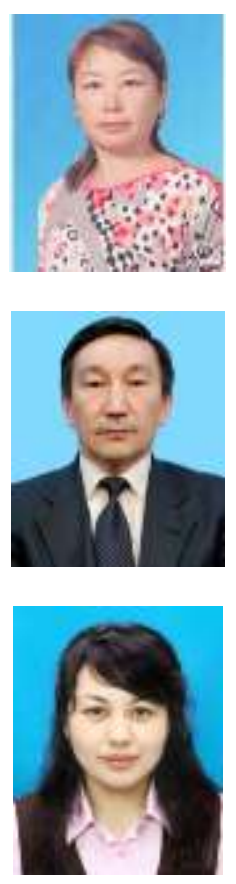

Zhanar Kemelbekova received the degree of candidate of technical Sciences at The Institute of Informatics and management of the Ministry of education and science of the Republic of Kazakhstan, Almaty in 2010. Currently, he works as an associate Professor of the Department of Informatics and Deputy Dean for scientific work of the Natural science and pedagogical Higher school of the South Kazakhstan state University M.Auezov. He has more than 80 scientific publications in the field of mathematical modeling, computer modeling, computer networks, computers and systems.

Ordabay Sembiev received a $\mathrm{PhD}$ in physics and mathematics from the Moscow aviation Institute in 1992. Then in 2007 he received the degree of doctor of technical Sciences in Kazakhstan. Currently, he works as a Professor at the South Kazakhstan state University.M. Auezov. He published more than 120 in the cited journals and conferences in the area of Mathematical and Computer modeling and Computer science. His main research interests are in the fields of Mathematical modeling and Computer Science.

Zhanat Umarova received the $\mathrm{PhD}$ degree in Computer Science from Kazakh National Technical University, Almaty in 2012. Currently she is working as an associated Professor of Information Systems department at M. Auezov South Kazakhstan State University. Her research interests include mathematical modeling, computer simulation, information security and data protection in information systems, renewable recourses. 